\title{
Eyewall Evolution of Typhoons Crossing the Philippines and Taiwan: An Observational Study
}

\author{
Kun-Hsuan Chou ${ }^{1, *}$, Chun-Chieh $\mathrm{Wu}^{2}$, and Yuqing Wang ${ }^{3}$ \\ ${ }^{1}$ Department of Atmospheric Sciences, Chinese Culture University, Taipei, Taiwan \\ ${ }^{2}$ Department of Atmospheric Sciences, National Taiwan University, Taipei, Taiwan \\ ${ }^{3}$ International Pacific Research Center, and Department of Meteorology, \\ University of Hawaii at Manoa, Honolulu, Hawaii, USA
}

Received 20 January 2011, accepted 10 May 2011

\begin{abstract}
This study examines the statistical characteristics of the eyewall evolution induced by the landfall process and terrain interaction over Luzon Island of the Philippines and Taiwan. Interesting eyewall evolution processes include the eyewall expansion during landfall, followed by contraction in some cases after re-emergence in warm ocean waters. Best track data, advanced satellite microwave imagers, high spatial and temporal ground-observed radar images and rain gauges are utilized to study this unique eyewall evolution process. An examination of the available microwave images of 23 typhoons crossing the Philippines between 2000 and 2010 shows that most typhoons experienced this kind of eyewall evolution, i.e., the radius of the eyewall for $87 \%$ of landfall typhoons increased during landfall; and, the radius of the eyewall for $57 \%$ of the cases contracted when the typhoons reentered the ocean after they crossed the Philippines. Furthermore, analyses of large-scale environmental conditions show that small vertical wind shear, high low-level relative humidity and sea surface temperature are important for the reorganization of the outer eyewall and the subsequent eyewall contraction when the typhoons reentered the ocean.

For typhoons that crossed Taiwan, based on the microwave and radar images, $89 \%$ of the cases show an expansion of the eyewall during landfall. However, the reorganization of the outer eyewall and subsequent contraction were rarely observed due to the small fraction of the ocean when the typhoons entered the Taiwan Strait. In addition, observed rainfall shows an expansion in the rainfall area induced by terrain.
\end{abstract}

Key words: Eyewall contraction, Eyewall breakdown, Eyewall reorganization

Citation: Chou, K. H., C. C. Wu, and Y. Wang, 2011: Eyewall evolution of typhoons crossing the Philippines and Taiwan: An observational study. Terr. Atmos. Ocean. Sci., 22, 535-548, doi: 10.3319/TAO.2011.05.10.01(TM)

\section{INTRODUCTION}

The eyewall of a tropical cyclone (TC) consists of deep convective clouds surrounding the eye that contains only light winds and almost no precipitation. The most severe weather, such as maximum winds and torrential rains, occurs in the eyewall. It is generally believed that the evolution of the eyewall is responsible for the intensity change of a TC. Therefore, the evolution of the eyewall has always been an intriguing issue in TC research. Recent studies have shed new insight into the internal thermodynamics and dynamics of TCs, such as the description of eyewall features

\footnotetext{
* Corresponding author

E-mail:zkx@faculty.pccu.edu.tw
}

and eye thermodynamics (Shapiro and Willoughby 1982; Willoughby 1998); the replacement of concentric eyewall (Willoughby et al. 1982; Willoughby and Black 1996); asymmetric mixing of the eye and eyewall in the formation of annular hurricanes (Knaff et al. 2003); the formation of mesovortices and the polygonal eyewall structure which are linked to barotropic instability and the potential vorticity mixing processes between the eye and the eyewall (Schubert et al. 1999; Kossin and Eastin 2001; Kossin and Schubert 2001); and the presence of convectively coupled vortex Rossby waves in the eyewall (Montgomery and Kallenbach 1997; Wang 2002). 
Another important factor affecting the eyewall evolution is the topography that a TC encounters. Brand and Blelloch (1973) was the first to document the effect of the Philippine Islands on the changes in the track, eye diameter, storm intensity and size of TCs during 1960 - 70 based on the Annual Typhoon Reports of Joint Typhoon Warning Center (with aircraft reconnaissance available during that period). It was indicated that the frictional effect of land mass and the reduction in heat and moisture supply by the ocean are the primary causes of the above changes (Wu et al. 2009).

There have been limited numbers of studies focusing on the eyewall dynamics of typhoons making landfall at the Philippine Islands, whose terrain has horizontal scales about equivalent to the size of the TC. The eyewall evolution of Typhoon Zeb (1998) before, during, and after its landfall at Luzon was documented from both the satellite observation and numerical simulation (Wu et al. 2003). It is proposed that the terrain plays a critical role in such an eyewall evolution wherein first the eyewall contraction just before landfall, its breakdown after landfall, and then the reorganization of a larger outer eyewall after the storm returns to the ocean. Further detailed numerical examination of this unique eyewall evolution was documented in Wu et al. (2009). It was shown that the presence of Luzon plays a critical role in the observed eyewall evolution. The eyewall replacement occurred in Typhoon Zeb was triggered by the mesoscale terrain that has a horizontal scale similar to the core of the typhoon. Furthermore, based on several sensitivity experiments, it was shown that both diabatic heating and surface friction are key factors for the maintenance and the reorganization of the outer eyewall when Zeb reentered the ocean.
In addition to Typhoon Zeb (1998) over Luzon, similar scenarios have also been observed in other storms, such as Typhoon Dan (1999) over Luzon, Typhoon Talim (2005) over Taiwan, and Hurricane Gilbert (1988), Wilma (2005) and Dean (2007) over the Yucatan Peninsula (Chou and Wu 2008). Figure 1 shows the topography of the Philippines and Taiwan. It can be found that both the Philippines and Taiwan have terrain features with horizontal scales (several hundred $\mathrm{km}$ ) similar to the size of TCs. In addition, it is shown that the area of the Philippines is wider than the island of Taiwan, and high mountains are located in the northern Philippines (Luzon island) while similar topography is found in central Taiwan. Since any changes in the eyewall structure would lead to or be accompanied by change in the storm intensity, it is believed that a better understanding of such terrain-induced eyewall evolution could improve our understanding of a TC structure and intensity changes, especially for TCs making landfall and interacting with mesoscale terrains (Wang and Wu 2004). Furthermore, the question of intensity and inner-core structure change, and the associated precipitation pattern of a landfalling TC is very important for operational forecasts.

The main objective of this study is to document the statistical characteristics of the eyewall evolution induced by the landfall process and terrain interaction over Luzon Island of the Philippines and Taiwan based on advanced satellite microwave imagers and high spatial and temporal radar images. The difference between the typhoons with and without reorganization of the outer eyewall when typhoons reentered the ocean will be examined. Furthermore, the differences in eyewall evolutions between the typhoons crossing the Philippines and those crossing the Taiwan are also
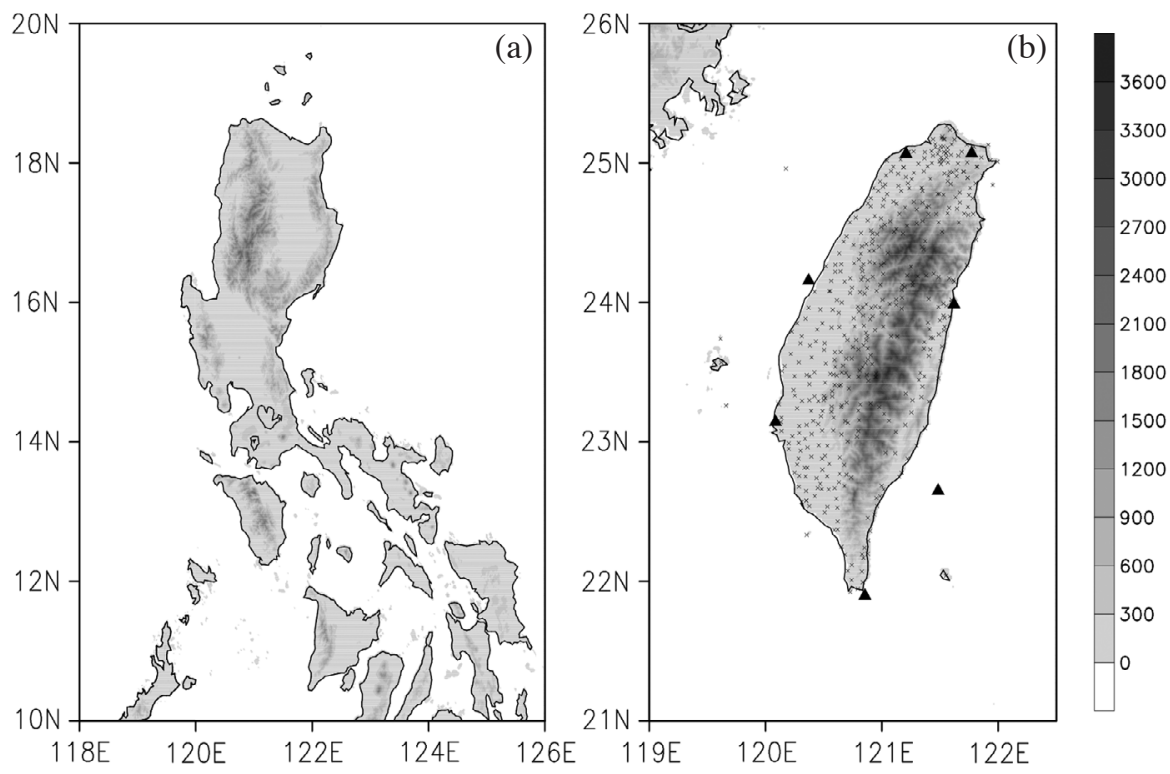

Fig. 1. The terrain height of 30-minute USGS (United States Geological Survey) digital elevation model data (unit: $\mathrm{m}$ ) of the Philippines (a) and Taiwan (b), respectively. The triangle and cross symbols in (b) represent the CWB radar and automatic rain gauge data stations. 
identified. Analysed data are described in section 2. The statistical characteristics of the eyewall evolution of typhoons crossing the Philippines and Taiwan are discussed in sections 3 and 4, respectively. The overall findings are summarized in the last section.

\section{THE DATA DESCRIPTIONS}

\subsection{Best Track TC Data}

Best track data including 6-hourly estimates of position and intensity are obtained from the Joint Typhoon Warning Center (JTWC). JTWC data are utilized to document the overall statistics for TCs crossing the Philippines during the period $1945-2010$. Since the eyewall structure is well organized only in strong TCs and that its evolution can be depicted using microwave imagers, this study will focus primarily on the eyewall evolution of TCs with maximum sustained surface winds larger than 64 knots (namely, "typhoons" as defined in this study).

\subsection{Satellite Microwave Imagery}

Microwave imagery allows for identification of rain and ice particle patterns within TC rainbands that are normally blocked by mid- to upper-level clouds in the infrared and visible imagery (Velden et al. 2006). Passive microwave digital data and imagery can provide key storm structural details and offset many of the visible/infrared spectral problems (Hawkins et al. 2001). The microwave imagers used in this study include the Special Sensor Microwave Imager (SSM/I), the Special Sensor Microwave Imager Sounder (SSM/IS), Tropical Rainfall Measuring Mission Microwave Imager (TRMM/TMI), and Advanced Microwave Scanning Radiometer - Earth Observing System instrument on board the Aqua satellite (AMSR-E/Aqua). The advantage of these imagers is that the cirrus clouds are transparent while using the 85 - $91 \mathrm{GHz}$ channel. Therefore, an excellent view of the eye, eyewall, and rainband structure of TCs can be provided by the $85-91 \mathrm{GHz}$ brightness temperature and the size of the eyewall can be well captured and estimated by these microwave imagers. The archived microwave imagers from 2000 to 2010 are obtained from the Navy Research Laboratory's Tropical Cyclone Webpage (http://www.nrlmry.navy. mil/tc pages/tc home.html).

\subsection{Radar Reflectivity}

The composite radar reflectivity data are taken from the Central Weather Bureau (CWB) radar network for analyzing the eyewall evolution when typhoons cross Taiwan. The dataset has $0.0125^{\circ}$ latitude by $0.0125^{\circ}$ longitude horizontal resolution and 10 minute temporal resolution. Currently, the only archived composite radar reflectivity data after 2005 are available, thus only typhoons that crossed Taiwan from
2005 - 2010 are examined with the radar reflectivity data. The locations of CWB radar stations are denoted by triangle symbols in Fig. 1b.

\subsection{NCEP FNL Global Analysis}

The National Centers for Environmental Prediction (NCEP) final gridded analysis (FNL) datasets are on $1.0^{\circ}$ latitude by $1.0^{\circ}$ longitude grids prepared operationally every six hours $(0000,0600,1200$ and 1800 UTC). The dataset is not a re-analysis product, but is obtained from the NCEP Global Data Assimilation System, which continuously collects observational data from the Global Telecommunications System and other sources. The analyses are available on the surface and at 26 mandatory (and other pressure) levels from 1000 to $10 \mathrm{hPa}$. Parameters include surface pressure, sea level pressure, geopotential height, temperature, sea surface temperature, soil values, ice cover, relative humidity, zonal and meridional velocity, vertical velocity, vorticity and ozone concentration (http://www.ncep.noaa. gov/).

In this study the environmental low level relative humidity and vertical wind shear near the core of TCs are calculated based on the NCEP FNL for each typhoon that crossed the Philippines during 2000 - 2010. The low-level (at $700 \mathrm{hPa}$ ) relative humidity is averaged over a $5^{\circ}$ latitudelongitude square, centered on the best-track location of each storm. The vertical wind shear was estimated based on the vector difference between the 200 - and $850-\mathrm{hPa}$ winds, averaged within a $2-8$ degree radius of the storm center. The method of shear calculation is determined by referring to Knaff et al. (2005) to keep the shear values from being corrupted by the bogus vortex in the global analysis.

\subsection{Sea Surface Temperature}

The NCEP Real-Time Global Sea Surface Temperature analysis (RTG-SST) has $0.5^{\circ}$ latitude by $0.5^{\circ}$ longitude horizontal resolution. The operational RTG-SST product uses in situ (ship and buoy) and satellite-derived SST data (NOAA-16 SEATEMP retrievals) from the Naval Oceanographic Office Major Shared Resource Center (Thiébaux et al. 2003). In this study, the RTG-SST near the core of $\mathrm{TC}$, i.e, averaged over a $5^{\circ}$ latitude by $5^{\circ}$ longitude square, centered at the best-track location of each typhoon, is calculated for each typhoon that crossed the Philippines during 2001 - 2010. Note that since the RTG-SST data are not available until 2001, the RTG-SST calculation is not available for typhoons in 2000 .

\subsection{Automatic Rain Gauge Data}

The Central Weather Bureau (CWB) automatic rain gauge data are used for rainfall analysis for typhoons that 
crossed Taiwan from 2005 - 2010. More than five hundred stations were deployed around the island from 2005 - 2010, and the rainfall data are recorded hourly. The locations of CWB rain gauge stations are denoted by cross symbols in Fig. 1b.

\section{THE EYEWALL EVOLUTION SCENARIOS FOR TYPHOONS CROSSING THE PHILIPPINES}

Figure 2 is a histogram showing the number of tropical storm cases (with the maximum sustained surface winds between 34 and 63 knots) and typhoon cases (with the maximum sustained surface winds larger than 64 knots) with their centers passing through either the Philippines or Taiwan from 1945 to 2010. It can be found that during this period on average about 26 TCs occurred over the western North Pacific per year, with 8.7 in the tropical storm category and 16.9 reaching typhoon intensity. Figure 2 a shows that among all the TCs, on average each year about 2.2 tropical storms and 3.0 typhoons made landfall at and crossed the Philippines, which accounts for about $20 \%$ of the total number of TCs in the western North Pacific. Among all the TCs crossing Taiwan (Fig. 2b), about 0.7 tropical storms and 1.3 typhoons made landfall and crossed Taiwan respectively, which accounts for $8 \%$ of the total number of TCs in the western North Pacific. As compared to the results of TCs crossing the Philippines, the amount of landfall TCs in Taiwan is roughly reduced by half.

It is our interest to examine the terrain-induced eyewall evolution as mentioned in the introduction. The 85 $91 \mathrm{GHz}$ brightness temperature microwave images available from 2000 to 2010 are used to depict the eyewall evolution for storms prior to, during and after landfall, after passing through the Philippines and reentering the ocean. During the 11-year period, 23 typhoons passed through the Philippines. Most of the storms either curved northward or continued
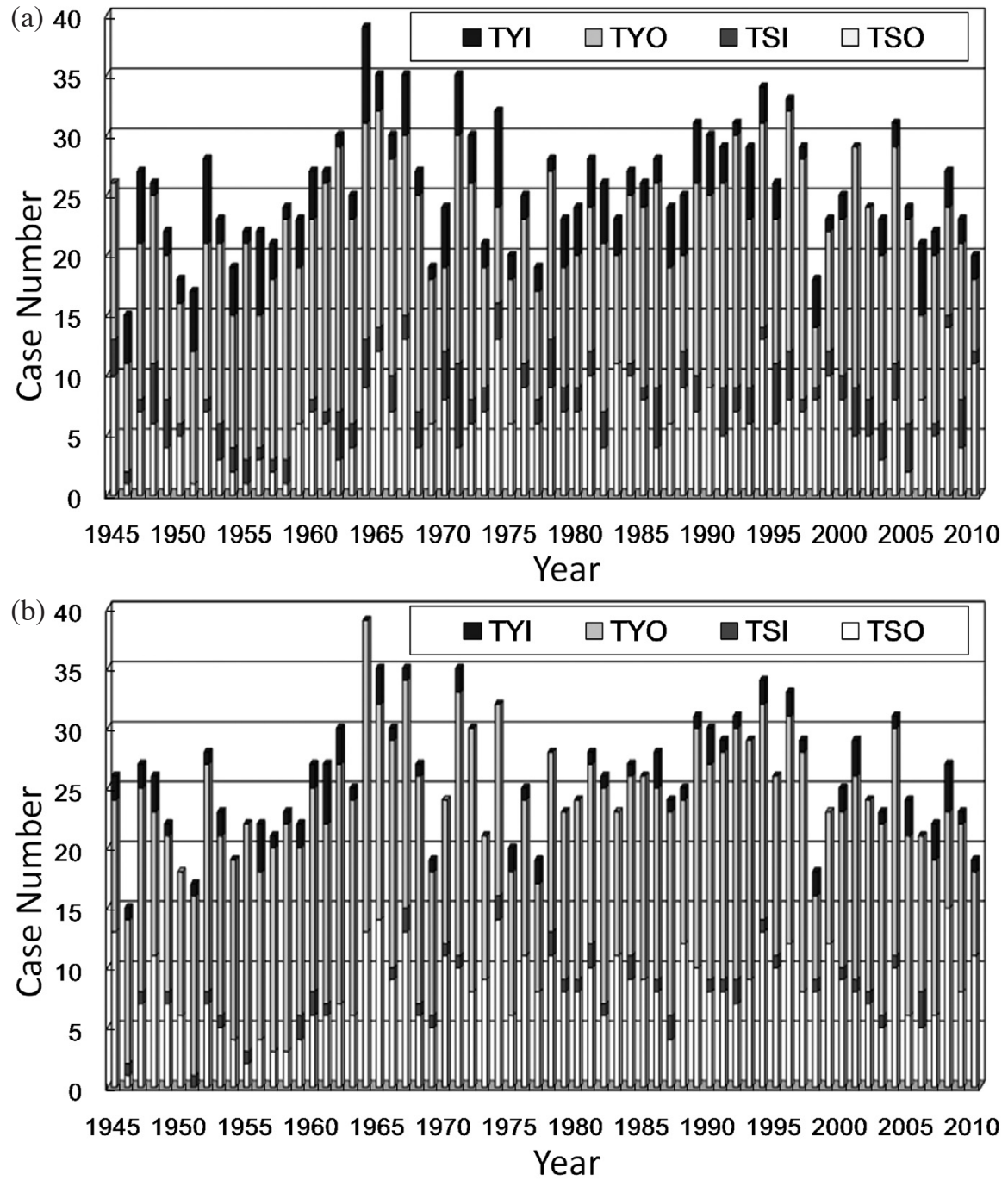

Fig. 2. Histogram of numbers of tropical storms (TS) and typhoons (TY) from JTWC best track from 1945 to 2010. TSI and TYI (TSO and TYO) indicate the tracks of TS and TY crossing (not crossing) the Philippines (a) and Taiwan (b), respectively. 
moving westward after crossing the Philippines. Later on, most of the typhoons affected Japan, Taiwan, China, and the Indochina Peninsula, respectively. The information about the duration time (the time each storm crossed the islands), intensity, and estimated radius of convective ring (eyewall) during the period when each storm crossed the Philippines is listed in Table 1.

Figure 3 shows the microwave images of the eyewall evolution before the storm made landfall, when it left the island, and after the storm reentered the ocean. Different characteristics are shown: three typhoons with reorganization of a larger outer eyewall (Xangsane in 2000, Cimaron in 2006, Megi in 2010), and one typhoon (Mitag in 2007) without reorganization of the outer eyewall. It can be found from these microwave images that the radii of the eyewalls generally reached a minimum before landfall, with radii of the eyewall ranging from 20 to $100 \mathrm{~km}$ (Figs. 3a, d, and g). After the storms left the Philippine islands, the eye became unidentifiable since the original primary eyewall convection weakened considerably due to the dissipation over the land/terrain and the reduction of the surface moisture supply (Figs. 3b, e, and h). In most (87\%) of the cases, the outer eyewall reappeared when the storms were about to leave the land and reenter the ocean. During this period, the radii of

Table 1. Information for the 23 selected typhoons that crossed the Philippines.

\begin{tabular}{|c|c|c|c|c|c|c|c|c|}
\hline TC NAME & DT & INTi & INTo & INTm & REi & REo & REr & OEC \\
\hline XANGSANE (2000) & 26 & 70 & 55 & 90 & $1-2$ & $2-3^{*}$ & $2^{* *}$ & Yes \\
\hline BEBINCA (2000) & 25 & 65 & 65 & 65 & 1 & $2^{*}$ & $1-2^{* *}$ & No \\
\hline IMBUDO (2003) & 7 & 130 & 80 & 90 & $1-2$ & $3-4^{*}$ & $2-3^{* *}$ & Yes \\
\hline KROVANH (2003) & 7 & 90 & 70 & 85 & $1-2$ & $2^{*}$ & 2 & No \\
\hline MELOR (2003) & 11 & 65 & 65 & 65 & $1-2$ & $2^{*}$ & 2 & No \\
\hline MUIFA (2004) & 18 & 115 & 60 & 90 & $1-2$ & $4^{*}$ & $2-3^{* *}$ & Yes \\
\hline NANMADOL (2004) & 4 & 130 & 100 & 100 & $1-2$ & $2-3^{*}$ & 4 & No \\
\hline ROKE (2005) & 20 & 80 & 40 & 40 & 1 & --- & --- & No \\
\hline CHANCHU (2006) & 36 & 75 & 75 & 125 & $1-2$ & $2-3^{*}$ & $2^{* *}$ & Yes \\
\hline XANGSANE (2006) & 24 & 65 & 100 & 115 & $1-2$ & $2-3^{*}$ & $2^{* *}$ & Yes \\
\hline CIMARON (2006) & 10 & 140 & 95 & 100 & 1 & $3-4^{*}$ & $2-3^{* *}$ & Yes \\
\hline CHEBI (2006) & 11 & 125 & 95 & 95 & $1-2$ & $2^{*}$ & $1-2^{* *}$ & No \\
\hline DURIAN (2006) & 20 & 135 & 95 & 95 & $1-2$ & $2^{*}$ & 2 & No \\
\hline UTOR (2006) & 23 & 65 & 90 & 90 & $1-2$ & $1-2$ & $1-2$ & No \\
\hline PEIPAH (2007) & 9 & 60 & 55 & 75 & 2 & $3-4^{*}$ & $2^{* *}$ & Yes \\
\hline MITAG (2007) & 5 & 95 & 65 & 65 & $2-3$ & $2-3$ & $2-3$ & No \\
\hline HALONG (2008) & 15 & 65 & 45 & 55 & $1-2$ & $2-3^{*}$ & $2-3$ & No \\
\hline FENGSHEN (2008) & 53 & 70 & 60 & 60 & 2 & $3-4^{*}$ & $3-4$ & No \\
\hline NURI (2008) & 7 & 95 & 90 & 90 & 2 & $4^{*}$ & $2-3^{* *}$ & No \\
\hline CHAN-HOM (2009) & 10 & 90 & 45 & 45 & $1-2$ & $2^{*}$ & $2-3$ & No \\
\hline PARMA (2009) & 12 & 135 & 65 & 65 & 2 & $3-4^{*}$ & $2-3^{* *}$ & No \\
\hline CONSON (2010) & 19 & 70 & 55 & 75 & $1-2$ & $2-3^{*}$ & $2^{* *}$ & Yes \\
\hline MEGI (2010) & 9 & 155 & 90 & 115 & $1-2$ & $3-4^{*}$ & $2-3^{* *}$ & Yes \\
\hline
\end{tabular}

Notes: INTi (INTo) represents the maximum sustained surface wind when the tropical storms moved in (out) of the islands and INTm represents the maximum sustained surface wind within $48 \mathrm{~h}$ after the storm left the islands. REi, REo, and REr mean the size of the major convective ring of the eyewall estimated by the satellite microwave images, for typhoons before landfall, while leaving the islands, and while reentering the ocean, respectively. Ring 1 indicates the area within 0.5 degree latitudes in radius from the storm center, and rings 2, 3, 4 represent the distance ranges of 0.5 - 1, 1 - 1.5, 1.5 - 2.0 degrees in radius, respectively. Superscript "*” means that the major convective ring expanded after landfall, and "**” denotes events in which the major convective ring contracted after reentering the ocean. OEC means the obvious reorganization of the outer eyewall when INTm is larger than INTo and REr is smaller than REo. Value of “---" indicates that the scenario is not identifiable. 

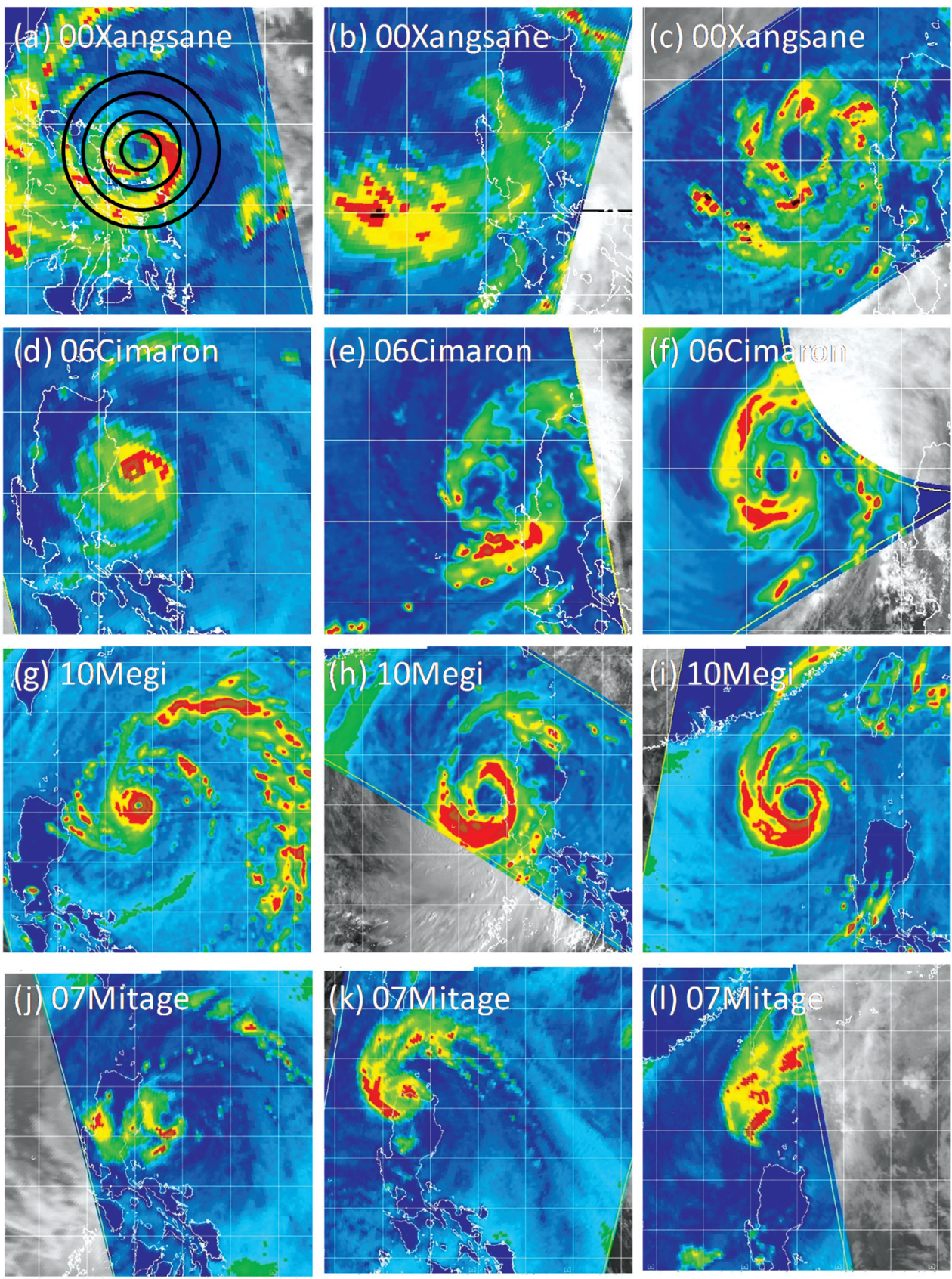

Fig. 3. The microwave images of 85 - $91 \mathrm{GHz}$ brightness temperature for three typhoons (Xangsane in 2000, Cimaron in 2006, and Megi in 2010), one with the outer eyewall reorganized and the other without (Mitag in 2007). Each case contains three individual images, showing the inner-core structure when typhoons moved into and away from the Philippines, and the reorganization of the larger outer eyewall when the storms reentered the ocean. The four rings shown in (a) are each within the range of $0-0.5,0.5-1,1-1.5,1.5-2.0$ degrees from the storm center.

the outer eyewall were roughly between 100 and $200 \mathrm{~km}$. After the storm reentered the ocean, the inner eyewall vanished and the outer eyewall was organized and contracted with radii between 50 and $150 \mathrm{~km}$ (Figs. 3c, f, and i). According to the microwave image analyses for 23 typhoons, $57 \%$ of them experienced reorganization of the outer eyewall. The estimated radii of the eyewall as storms cross the Philippines are shown in the Table 1.
Considering the intensity evolution based on the besttrack data for typhoons passing through the Philippines (Table 1), $83 \%$ of the typhoons influenced by the topography weakened during landfall, while four of them $(17 \%)$ intensified because their tracks passed through a rather small fraction of the landmass in the southern part of the Philippine islands. When the typhoons left the islands and reentered the ocean, 11 of them $(48 \%)$ re-intensified. Note that these 
re-intensification cases are not exactly the same with reorganization of the outer eyewall depicted in the microwave images. Regarding both the intensity and eyewall evolutions, 9 typhoons (39\%) experienced both re-intensification and reorganization of the outer eyewall after they reentered the ocean. These cases are called the obvious reorganization eyewall cases, while the others are called the non-obvious reorganization eyewall cases.

Figure 4 shows the duration and environmental conditions of the obvious and non-obvious reorganization eyewall typhoons. The duration is defined by the time difference between the landfall of the storm and its exit from the island. The duration is random in the two groups (Fig. 4a). The mean duration of the obvious reorganization eyewall cases is 17.6 hours, which is slightly longer than the nonobvious reorganization eyewall cases of 16.0 hours. This is likely not statistically significant.

Comparisons of the environmental conditions between the obvious and non-obvious reorganization eyewall cases (Figs. 4b, c, and d) indicate that the former appears to be embedded in an environment with smaller vertical wind shear, higher low-level relative humidity, and higher sea surface temperature than the later. The differences in mean vertical wind shear, low-level relative humidity, and sea surface temperature between the obvious and non-obvious reorganization eyewall cases are $-3.0 \mathrm{~m} \mathrm{~s}^{-1}, 6.8 \%, 1.2^{\circ} \mathrm{C}$, respectively. Note that the above results are based on 23 typhoons crossing the Philippine islands in the past 11 years, thus making them statistically less significant. However, these three environmental factors influencing the reorganization of the outer eyewall are rather consistent with the necessary conditions of tropical cyclone genesis proposed by Gray (1968), i.e., the favorable vertical shear pattern, moist midtroposphere, and warm SST.

\section{THE EYEWALL EVOLUTION SCENARIOS FOR TYPHOONS CROSSING TAIWAN}

As shown in Fig. 2b, there were 19 typhoons crossing Taiwan from 2000 - 2010. The same microwave image analyses are applied to these typhoons. Figure 5 shows six particular typhoons (Talim in 2005, Sepat and Krosa in 2007, Jangmi in 2008, Morakot in 2009, and Fanapi in 2010) with smaller eyewalls before landfall and larger
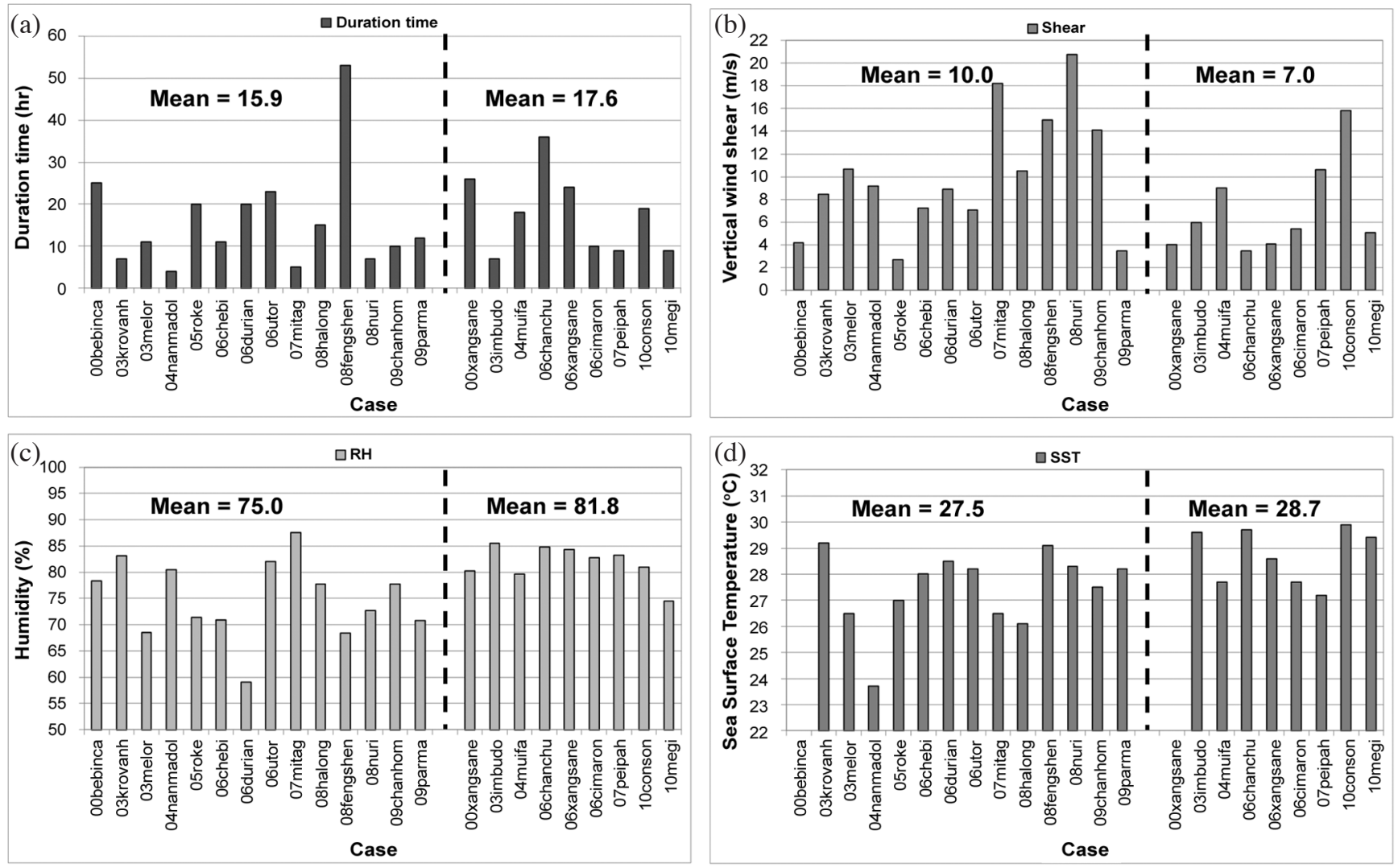

Fig. 4. The duration (a), environmental vertical wind shear (b), low-level relative humidity (c), and sea surface temperature (d) for TY cases which crossed the Philippines in 2000 - 2010. The vertical wind shear is averaged between a 2 to 8 degree radius from the storm center and averaged within $48 \mathrm{~h}$ after the storm left the islands. The low level relative humidity is averaged in a $5^{\circ} \times 5^{\circ}$ box centered at the storm center and averaged within $48 \mathrm{~h}$ after the storm left the islands. The sea surface temperature is also calculated as the mean in a $5^{\circ} \times 5^{\circ}$ box centered at the storm center at the same time before the typhoon made landfall. 
(a) 05Talim

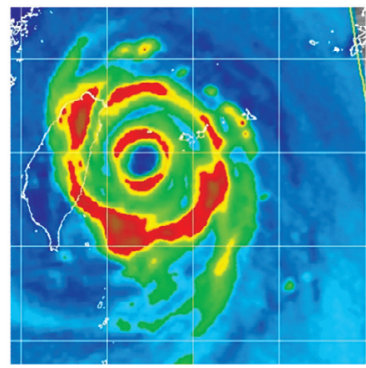

(e) $07 \mathrm{krosa}$

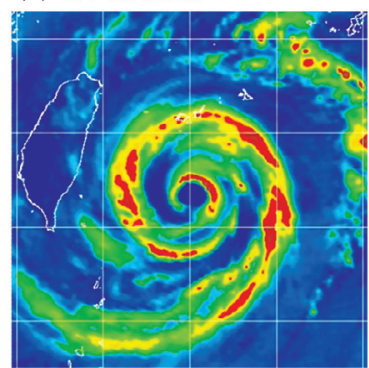

(i) 09Morakot

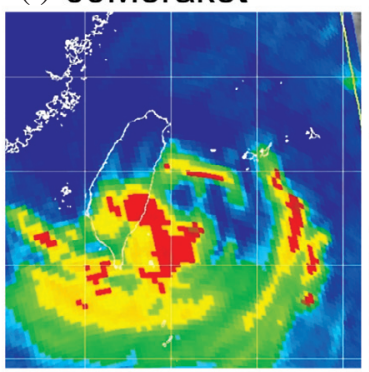

(b) 05Talim

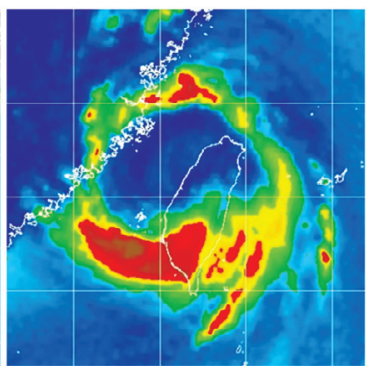

(f) $07 \mathrm{krosa}$

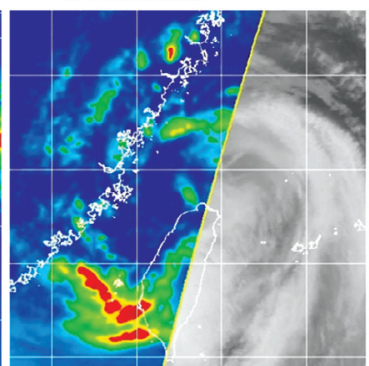

(j) 09Morakot

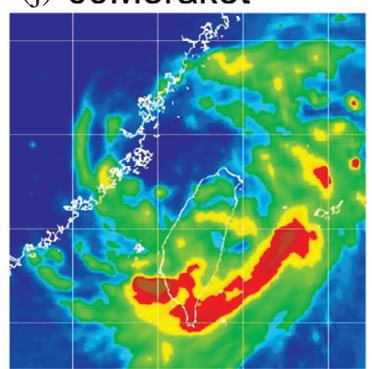

(c) 07Sepat

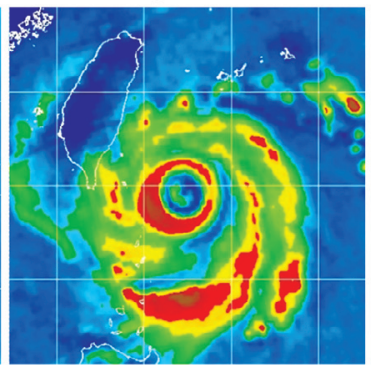

(g) 08Jangmi

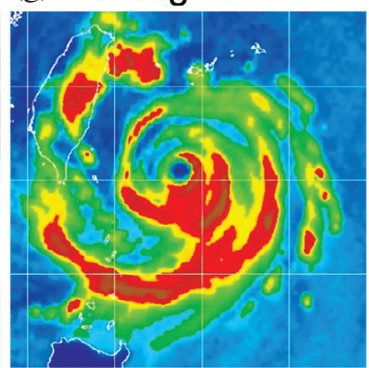

(k) 10Fanapi

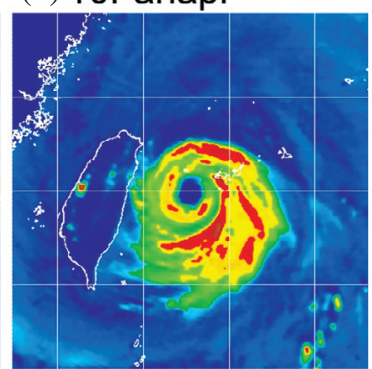

(d) 07Sepat

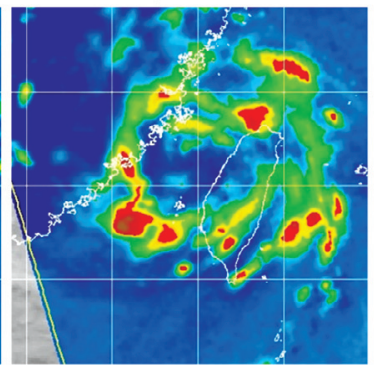

(h) 08Jangmi

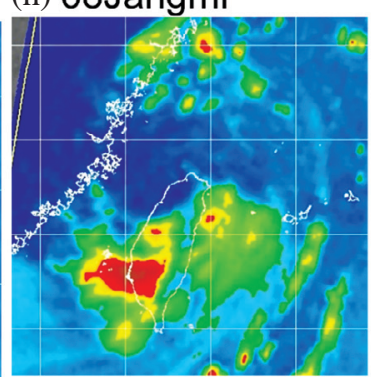

(l) 10Fanapi

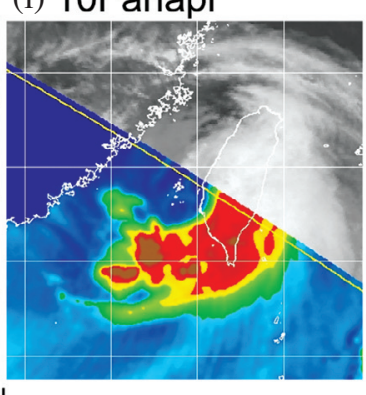

Naval Research Lab www.nrlmry.navy.mil/sat_products.html $<--\quad 85 \mathrm{H}$ Brightness Temp (Kelvin) -->

190 200 210 230 240 250 260 270

Fig. 5. Same as in Fig. 3, but for six typhoons (Talim in 2005, Sepat and Krosa in 2007, Jangmi in 2008, Morakot in 2009, and Fanapi in 2010) that crossed Taiwan. Each case contains two individual images, showing the inner-core structure changes when typhoons moved into and away from Taiwan.

eyewalls after entering the Taiwan Strait. Note that for typhoons crossing Taiwan, reorganization of the outer eyewall is rarely observed because the storms generally pass through the Taiwan Strait and make landfall over Mainland China within short periods of time (usually less than 36 hours). Information about the duration, intensity, and estimated radius of the convective eyewall during the period of each storm crossing Taiwan are shown in Table 2. The minimum and maximum duration of typhoons crossing Taiwan are 1.0 and $46.0 \mathrm{~h}$, respectively, with an average of $12.7 \mathrm{~h}$. In terms of intensity evolution, all typhoons weakened during the landfall, except in 2 cases (Nari and Lekima in 2001) the typhoon intensified after entering the Taiwan Strait. With regard to the evolution of the eyewall convection, with the exception of Kaitak in 2000 and Mindulle in 2004 , in about $89 \%$ cases, the major convective ring shifted from the central area to the outer area of the storm.
Figure 6 shows the radar reflectivity evolutions of the same six typhoons crossing Taiwan. Following the subjective definition of the outer eyewall by Kossin and Sitkowski (2009), the major convective ring is identified and plotted in the figure when there is at least $75 \%$ of a complete circular band with 0.5 degree width. Taking Typhoon Talim in 2005 as an example (Figs. 6a and b), the major convective ring before landfall and after the storm reentered the ocean was located at the radii of 0.6 and 1.7 degree latitude from the storm center, respectively. Based on these radar images, the convective rings are usually located within a 1.0 degree radius from the storm center before landfall, and they shift to the outer area ranging between 1.0 and 2.0 degree radii from the storm center after reentering the ocean. In the cases of Talim, Sepat, Krosa, Jangmi, Morakot, and Fanapi, the landfall of the typhoons in Taiwan lead to increment of the radius of the major convective ring by $0.9,0.7,1.4,1.2,1.4$, and 
0.2 degrees, respectively. These results are consistent with the eyewall evolution as depicted by the microwave images in Fig. 5, while a quantitative analysis is preformed based on radar images with high temporal and spatial resolution.

Detailed evaluations of the eyewall evolution for typhoons crossing Taiwan are analyzed by the radius-time Hovmöller diagrams of the azimuthally-averaged radar reflectivity as shown in Fig. 7. It can be found that most typhoons (58\%) showed a contraction of the inner eyewall and the presence of concentric eyewall before landfall over Taiwan (Figs. 7a, b, e, f, h, j, and 1). The inner eyewalls in most cases are located within a 1.0 degree radius from the storm center, while the outer eyewalls are located at a radius about $1.0-2.0$ degrees from the storm center. Both the inner and outer eyewalls obviously collapsed, and the convection areas shifted inward at the beginning of the landfall. Thereafter, the inner eyewall gradually weakened and the outer eye- wall reorganized several hours later after the storms made landfall. The radii of the reorganized outer eyewall range were between $1.0-2.2$ degrees, with the smallest and largest being in Pabuk in 2007 and Haitang in 2005 (as shown in Figs. 7d and a). Note that except for Sinlaku, Jangmi, and Fanapi, the reorganized eyewall did not contract when the storms cross the Taiwan Strait.

Overall, results from these Hovmöller-diagram analyses are not only consistent with those depicted by the microwave images, but also provide a high temporal and spatial observational evidence in demonstrating the terrain-induced eyewall evolution when typhoons cross islands as indicated earlier in Wu et al. (2003). Furthermore, the contraction of the eyewall before the storms made landfall at Taiwan was also consistent with the observational analyses (Willoughby and Black 1996; Chang et al. 2009) and the numerical simulations (Wu et al. 2009).

Table 2. Same as in Table 1, but for typhoons that crossed Taiwan.

\begin{tabular}{|c|c|c|c|c|c|c|c|c|}
\hline TC NAME & DT & INTi & INTo & INTm & REi & REo & REr & OEC \\
\hline KAITAK (2000) & 5 & 65 & 55 & 55 & $2-3$ & --- & --- & --- \\
\hline BILIS (2000) & 5 & 140 & 120 & 120 & $1-2$ & $4^{*}$ & --- & --- \\
\hline TORAJI (2001) & 11 & 95 & 75 & 75 & $1-2$ & $2^{*}$ & --- & --- \\
\hline NARI (2001) & 46 & 85 & 30 & 55 & $1-2$ & $2^{*}$ & --- & --- \\
\hline LEKIMA (2001) & 28 & 90 & 25 & 35 & 2 & $4^{*}$ & --- & --- \\
\hline MORAKOT (2003) & 8 & 65 & 60 & 60 & 2 & $2-3^{*}$ & --- & --- \\
\hline MINDULLE (2004) & 11 & 65 & 45 & 45 & $3-4$ & $2-3$ & --- & --- \\
\hline HAITANG (2005) & 11 & 95 & 75 & 75 & 2 & $2-3^{*}$ & --- & --- \\
\hline TALIM (2005) & 6 & 110 & 70 & 70 & 2 & $4^{*}$ & --- & --- \\
\hline LONGWANG (2005) & 5 & 115 & 90 & 90 & $1-2$ & $2-3^{*}$ & --- & --- \\
\hline PABUK (2007) & 1 & 70 & 65 & 65 & $1-2$ & $1-2^{*}$ & --- & --- \\
\hline SEPAT (2007) & 6 & 105 & 85 & 85 & 2 & $3-4^{*}$ & --- & --- \\
\hline KROSA (2007) & 3 & 115 & 90 & 90 & $1-2$ & $4^{*}$ & --- & --- \\
\hline KALMAEGI (2008) & 10 & 90 & 55 & 55 & $1-2$ & $3-4^{*}$ & --- & --- \\
\hline FUNGWONG (2008) & 17 & 95 & 70 & 70 & 2 & $3^{*}$ & --- & --- \\
\hline SINLAKU (2008) & 26 & 100 & 65 & 65 & 2 & $2-3^{*}$ & --- & --- \\
\hline JANGMI (2008) & 21 & 115 & 60 & 60 & $2-3$ & $3-4^{*}$ & --- & --- \\
\hline MORAKOT (2009) & 11 & 80 & 45 & 45 & 2 & $4^{*}$ & --- & --- \\
\hline FANAPI (2010) & 10 & 105 & 65 & 70 & 2 & $3-4^{*}$ & --- & --- \\
\hline
\end{tabular}

Notes: INTi (INTo) represents the maximum sustained surface wind when the tropical storms moved in (out) of the islands and INTm represents the maximum sustained surface wind within $48 \mathrm{~h}$ after the storm left the islands. REi, REo, and REr mean the size of the major convective ring of the eyewall estimated by the satellite microwave images, for typhoons before landfall, while leaving the islands, and while reentering the ocean, respectively. Ring 1 indicates the area within 0.5 degree latitudes in radius from the storm center, and rings 2, 3, 4 represent the distance ranges of $0.5-1,1-1.5,1.5-2.0$ degrees in radius, respectively. Superscript “*” means that the major convective ring expanded after landfall, and "**" denotes events in which the major convective ring contracted after reentering the ocean. OEC means the obvious reorganization of the outer eyewall when INTm is larger than INTo and REr is smaller than REo. Value of "---" indicates that the scenario is not identifiable. 

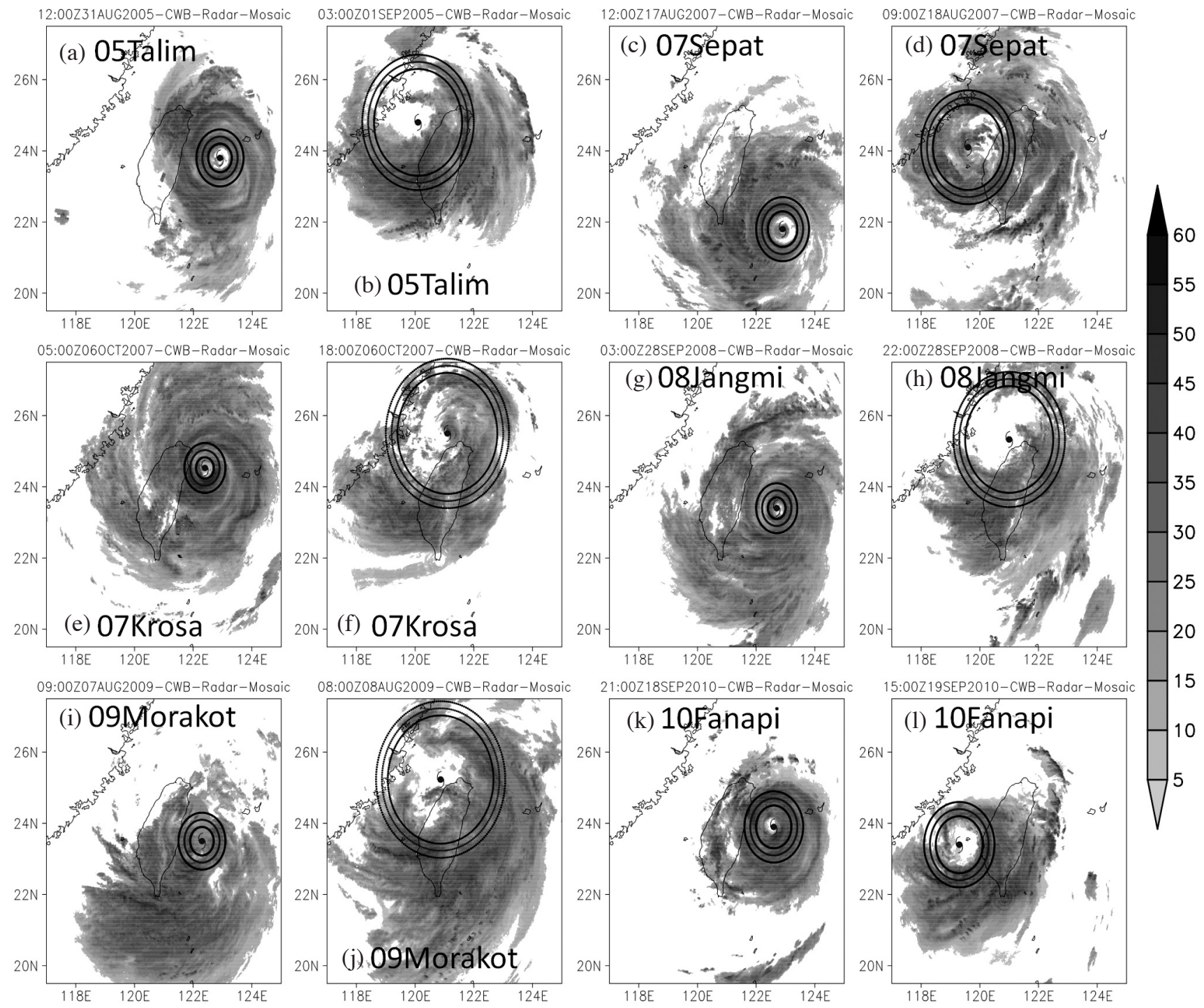

Fig. 6. The radar reflectivity evolutions of six particular typhoons (Talim in 2005, Sepat and Krosa in 2007, Jangmi in 2008, Morakot in 2009, and Fanapi in 2010) that crossed Taiwan. Each case contains three individual images, showing the inner-core structure changes when typhoons moved into and away from Taiwan. Three solid rings with a 0.5 degree radius are shown for identifying the maximum convection regions near the core of the typhoons. The locations of typhoons are interpolated from 3-h best track of CWB.

Figure 8 shows the radius-time Hovmöller diagrams of the azimuthally-averaged (relative to the storm center) rainfall rate observed hourly for typhoons crossing Taiwan during 2005 - 2010. The azimuthally-averaged rainfall rate is calculated from the storm center to a 5 degree radius with a bin size of a 0.1 degree radius. It should be noted that since the frequency of rainfall data is observed hourly, the distance between each rain gauge and the location of the storm center is calculated every hour, while the hourly location of the storm center is interpolated from 3-hourly CWB best track. It can be found that before the landfall and first several hours during the landfall period, except for Pabuk in 2007 and Kalmaegi in 2008 (Figs. 8d and g), the areas with larger rainfall rate were always located within a 0.5 degree radius from the storm center. Then, the rainfall rate in the storm core gradually vanished and the locations of the maximum rainfall rate shifted outward to a $1.0-2.0$ degree radius from the storm center when the storms left Taiwan. These results are consistent with the analyses of the radiustime Hovmöller diagrams of radar reflectivity. Furthermore, these rainfall analyses indicate that the topography of Taiwan not only alters the evolution of the eyewall, but also modifies the rainfall pattern of landfall typhoons. Note that Pabuk passed through the southern part of Taiwan in just one hour. Therefore, the modulation of the eyewall and rainfall pattern for Pabuk may not be as significant as the other cases. The unique rainfall patterns induced by Kalmaegi and Morakot are related to the confluent region associated with 


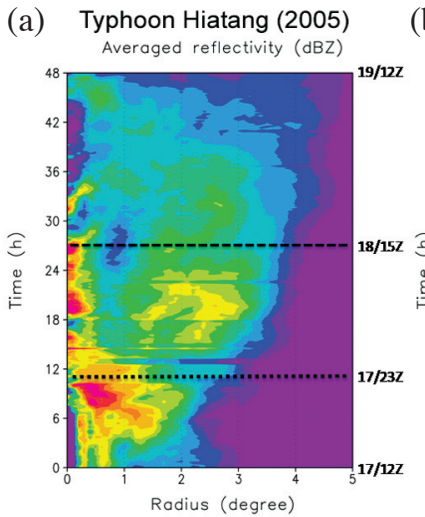

(e) Typhoon Sepat (2007)

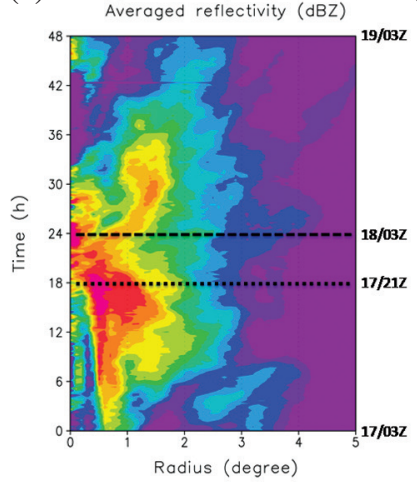

(i) Typhoon Sinlaku (2008)

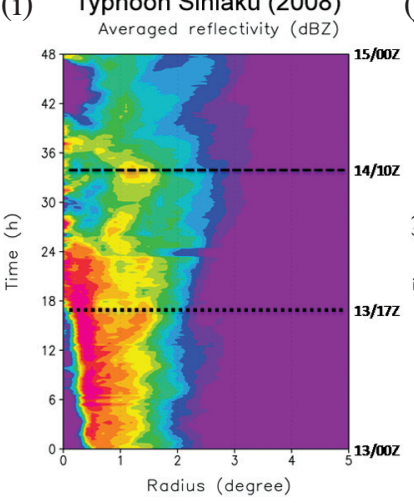

(b) Typhoon Talim (2005)

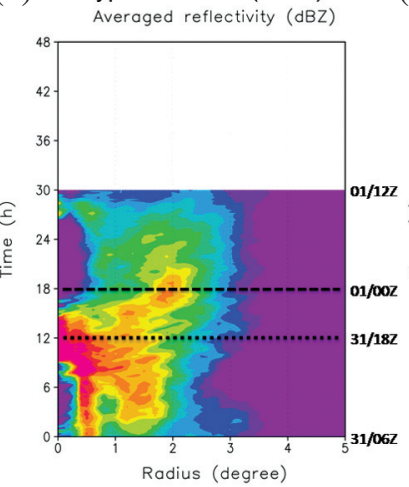

(f)

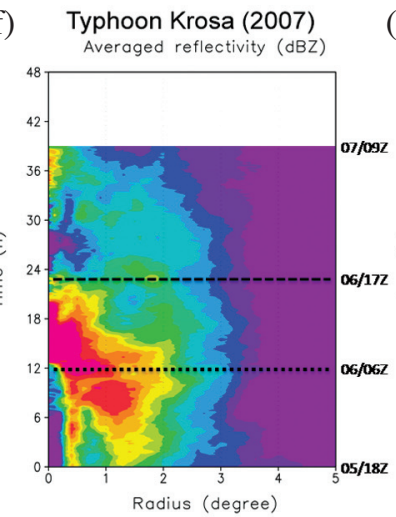

(j)

(j)

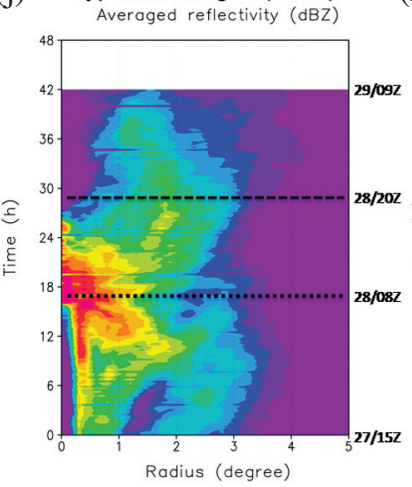

(c) Typhoon Longwang (2005)

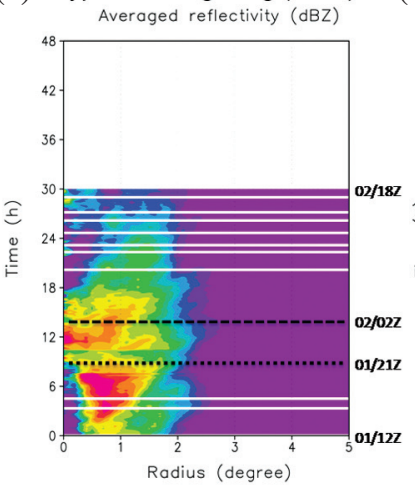

(g) Typhoon Kalmaegi (2008) Averoged reflectivity (dBZ)

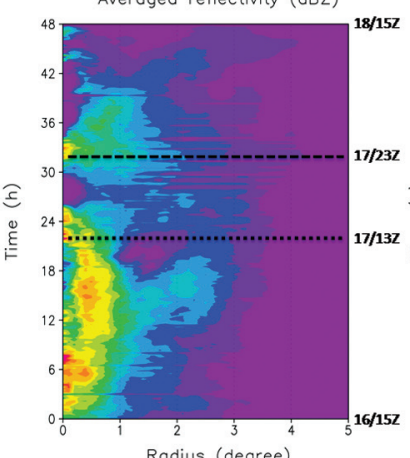

(k) Typhoon Morakot (2009)

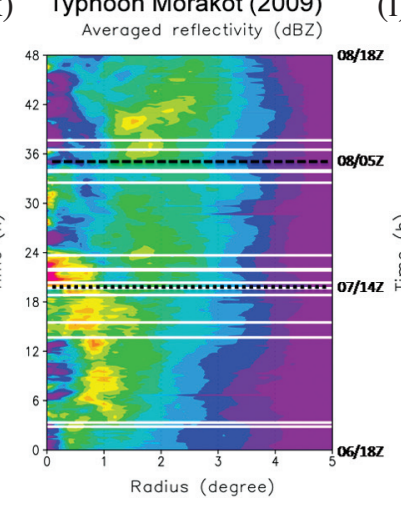

(h) (d) Typhoon Pabuk (2007)
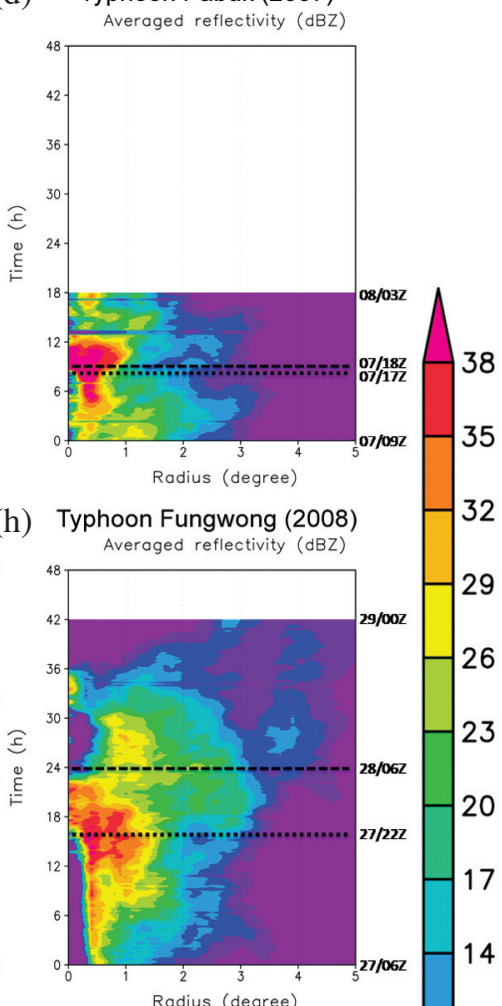

(1)

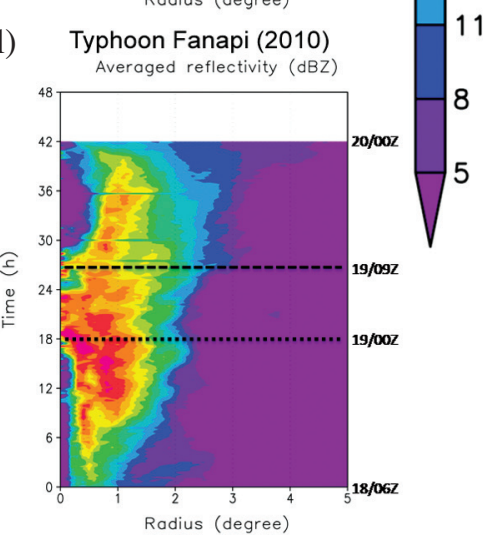

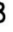

\section{s}

32

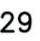

26

23

\section{0}

17

14

1

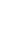

Fig. 7. Radius-time Hovmöller diagram of the azimuthally-averaged radar reflectivity (dBZ) for 12 typhoons that crossed Taiwan. The dotted (dashed) horizontal line indicates the time when typhoons made landfall at (exited from) Taiwan.

the storm circulation and the environmental southwesterly monsoon flows as proposed in Ge et al. (2010) and Hong et al. (2010). However, the rainfall pattern expanding outward from the storm center caused by the terrain effect is consistent with the process revealed in this study.

\section{CONCLUDING REMARKS}

The statistical characteristics of the terrain-induced eyewall evolution when a typhoon makes landfall, as in the discussion about Typhoon Zeb (1998) over Luzon Island by Wu et al. $(2003,2009)$, are examined based on the best track data, advanced satellite microwave imagers, high spatial and temporal ground-observed radar images and rain gauges in this study. Based on the analyses of JTWC best track data from 1945 - 2010, it is found that annually at least 3.0 TCs with sustained surface maximum winds larger than 64 knots passed through the Philippine islands and 1.3 through Taiwan. During the eleven-year period from 2000 and 2010, the eyewall evolution associated with the 23 and 19 typhoons respectively making landfall in the Philippine islands and Taiwan, were detected by the $85-91 \mathrm{GHz}$ brightness temperature satellite microwave imagers. Based upon these available microwave images for typhoons crossing the Philippine islands, the results indicate that the radius of the eyewall increased during landfall in $87 \%$ of the 

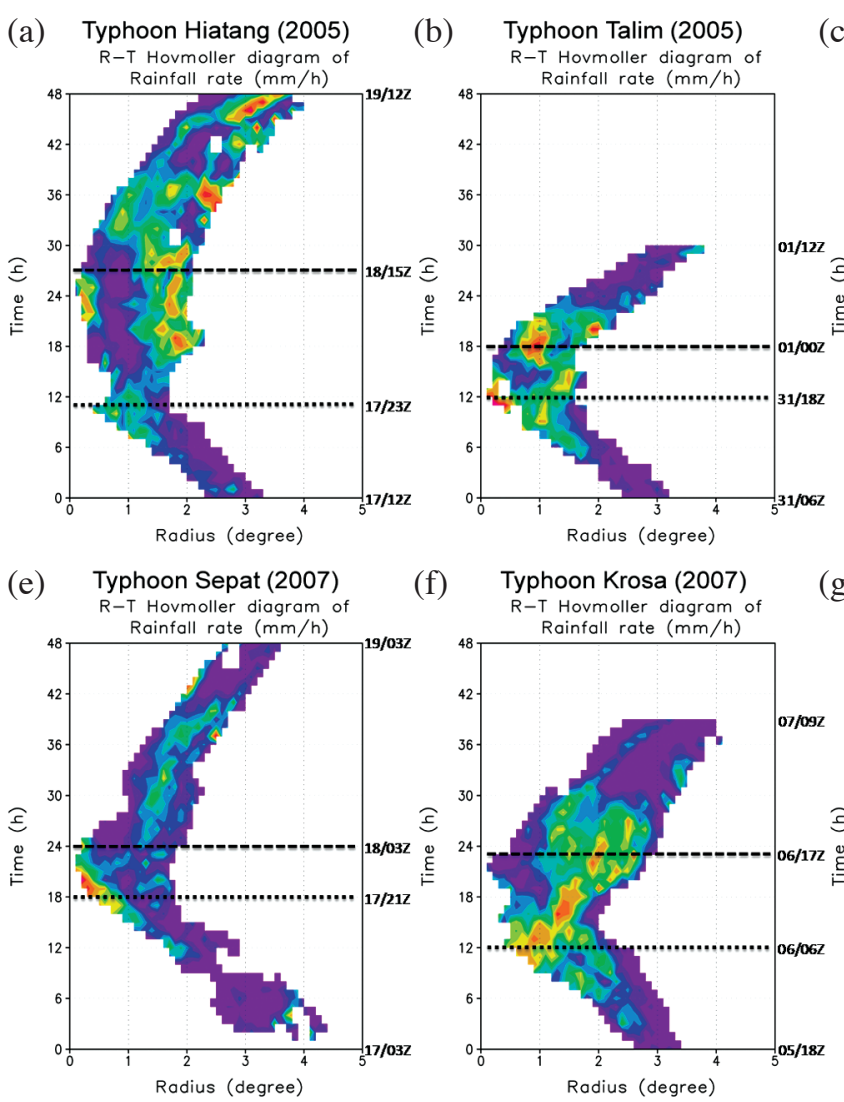

(i) Typhoon Sinlaku (2008)

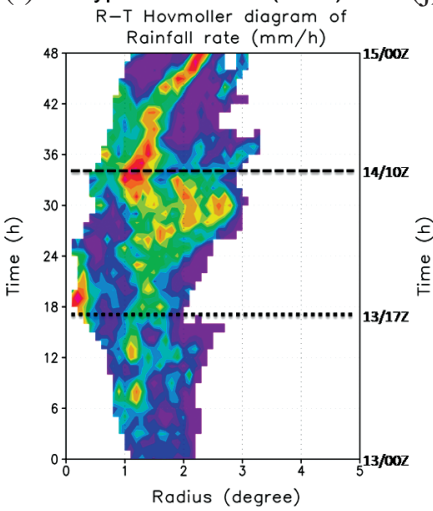

(f)

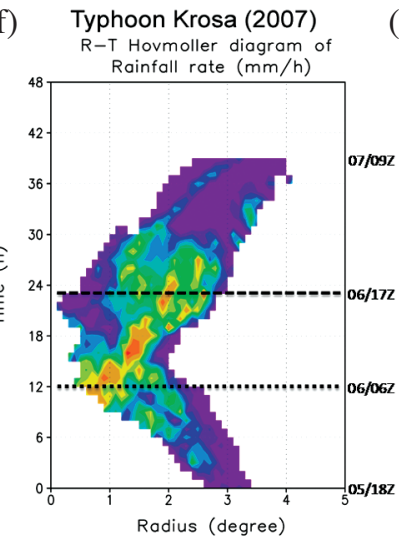

(j)

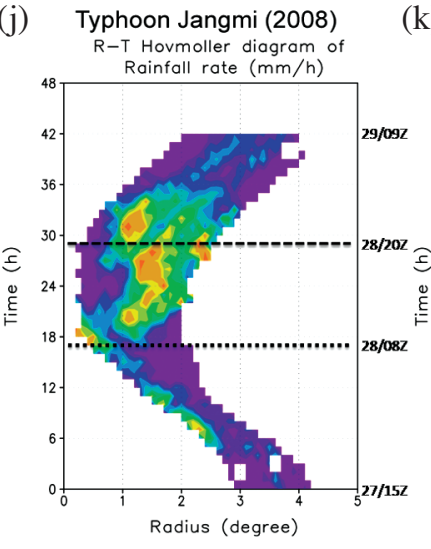

(c) Typhoon Longwang (2005) $\mathrm{R}-\mathrm{T}$ Hovmoller diagram of Rainfall rate $(\mathrm{mm} / \mathrm{h})$

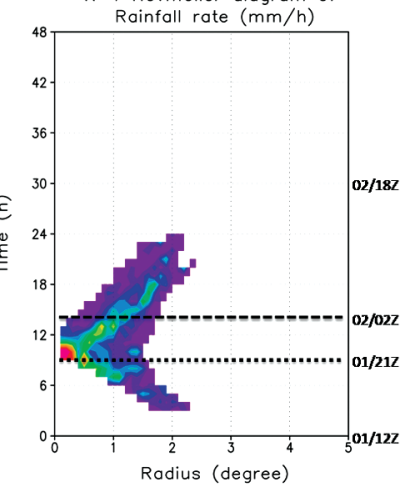

(g) Typhoon Kalmaegi (2008)

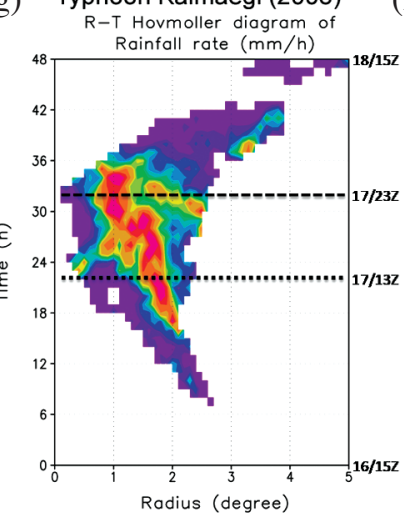

(k)
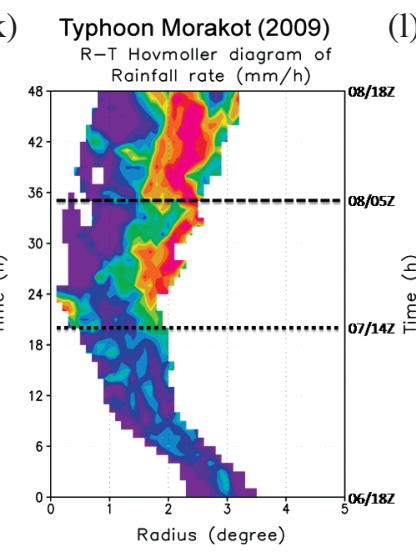

(1) (d) Typhoon Pabuk (2007)

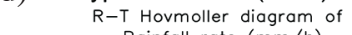
Rainfall rate $(\mathrm{mm} / \mathrm{h})$

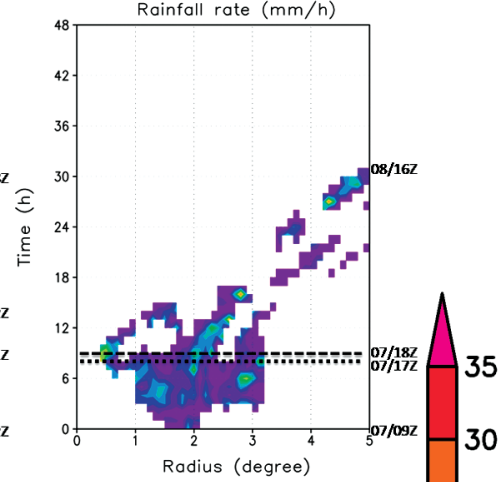

(h) Typhoon Fungwong (2008) 25
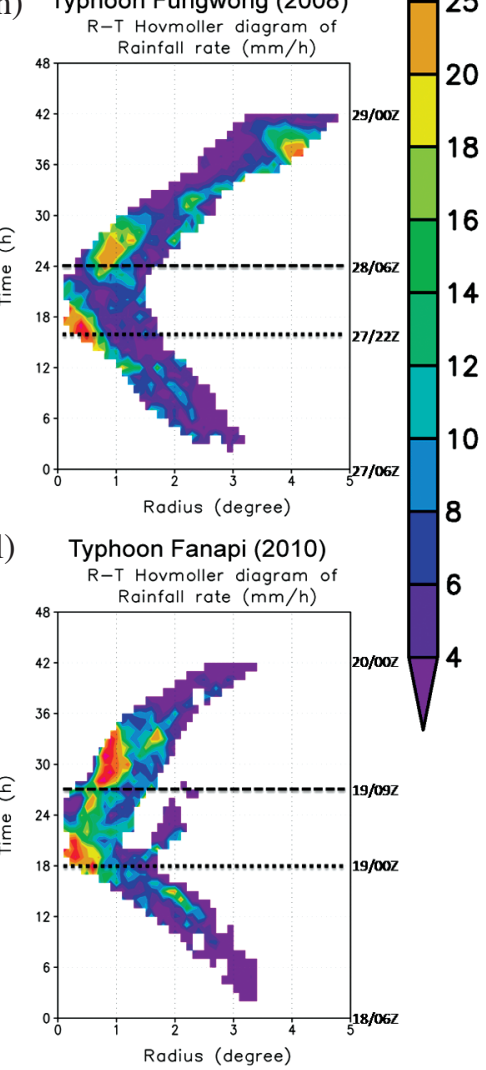

Fig. 8. Radius-time Hovmöller diagram of the azimuthally-averaged (relative to the storm center) observed rainfall rate ( $\mathrm{mm} \mathrm{h}^{-1}$ ) for 12 typhoons that crossed Taiwan. The dotted (dashed) horizontal line indicates the time when typhoons made landfall at (exited from) Taiwan. The observed rainfall rates are calculated from the CWB automatic rain gauge.

landfalling typhoons, while in $57 \%$ of the cases the radius of the eyewall contracted when the typhoon reentered the ocean. For typhoons crossing Taiwan, both the microwave images from satellites and the reflectivity images from ground-based radar observations are applied to analyze the eyewall evolution. It is found that $89 \%$ of cases show an expansion of the eyewall during the landfall period. However, reorganization of the outer eyewall is seldom observed due to the limited time the typhoon spends over the Taiwan Strait before making landfall in Mainland China. Furthermore, based on the observed rainfall analyses, an ex- pansion of the rainfall pattern due to the effect of terrain is also revealed. These results provide a conceptual model that may help improve the forecast of severe weather associated with such type of landfall storms. It should be noted that the findings of this study could provide more insights into the impact of terrain on rainfall caused by landfall TCs. In a traditional view, a landfall TC provides stronger wind on the windward side, which then enhances the orographic lifting, thereafter producing more rainfall (Wu and Kuo 1999). Large-scale environmental conditions are examined to investigate the differences between contracted and non- 
contracted outer eyewall cases. The results show that small vertical wind shear, high low-level relative humidity, and warm sea surface temperatures are important for the reorganization of the outer eyewall after the landfall typhoons reenter the ocean.

The eyewall evolution examined by the satellite microwave imagers is consistent with the finding by Brand and Blelloch (1973), who indicated that the islands of the Philippines affected the eye diameter of passing typhoons based on aircraft reconnaissance data. Although this special eyewall evolution had been identified by Brand and Blelloch (1973), we have documented the climatological aspects of such eyewall evolution and also shown that advanced satellite microwave imagers can depict the eyewall evolution for almost all cases selected. Furthermore, this study also shows that this special eyewall evolution can also be found in typhoons making landfall in Taiwan from high spatial and temporal radar images.

Although this terrain-induced eyewall evolution has been better documented, some issues remain unsolved and are yet to be addressed in future studies. (1) How is such an eyewall evolution influenced by the size of the land, the height of the terrain, and the size and intensity of the landfalling storm? (2) What determines the timing for the inner eyewall to break down after the storm makes landfall? (3) What is the relationship between land with different surface roughness, latent heat flux supply, and topography? (4) How does the environmental vertical wind shear affect this eyewall evolution? It is expected that more insight can be obtained from more well-designed sensitivity numerical experiments with high-resolution cloud-resolving models.

Acknowledgements The authors would like to express their sincere appreciation to Dr. P. L. Chang for providing the CWB radar mosaic data. The work is supported by the National Science Council of Taiwan through Grants NSC98-2111-M-034-002, NSC98-2111-M-002-008-MY3, and NSC-99-2111-M-034-004. YW has been supported in part by US NSF grant ATM-0754039.

\section{REFERENCES}

Brand, S. and J. W. Blelloch, 1973: Changes in the characteristics of typhoons crossing the Philippines. J. Appl. Meteorol., 12, 104-109, doi: 10.1175/1520-0450(1973) 012<0104:CITCOT>2.0.CO;2. [Link]

Chang, P. L., B. J. D. Jou, and J. Zhang, 2009: An algorithm for tracking eyes of tropical cyclones. Weather Forecast., 24, 245-261, doi: 10.1175/2008WAF2222112.1. [Link]

Chou, K. H. and C. C. Wu, 2008: Eyewall evolution for typhoons crossed the terrains. Preprints, $28^{\text {th }}$ Conference on Hurricanes and Tropical Meteorology, Orlando, FL, Amer. Meteor. Soc., 17C.2.
Ge, X., T. Li, S. Zhang, and M. Peng, 2010: What causes the extremely heavy rainfall in Taiwan during Typhoon Morakot (2009)? Atmos. Sci. Lett., 11, 46-50, doi: 10. 1002/asl.255. [Link]

Gray, W. M., 1968: Global view of the origin of tropical disturbances and storms. Mon. Weather Rev., 96, 669700, doi: 10.1175/1520-0493(1968)096<0669:GVOT OO $>2.0 . C O ; 2$. [Link]

Hawkins, J. D., T. F. Lee, J. Turk, C. Sampson, J. Kent, and K. Richardson, 2001: Real-time internet distribution of satellite products for tropical cyclone reconnaissance. Bull.Amer.Meteorol.Soc., 82, 567-578, doi: 10.1175/ 1520-0477(2001)082<0567:RIDOSP>2.3.CO;2. [Link]

Hong, C. C., M. Y. Lee, H. H. Hsu, and J. L. Kuo, 2010: Role of submonthly disturbance and 40-50 day ISO on the extreme rainfall event associated with Typhoon Morakot (2009) in southern Taiwan. Geophys. Res. Lett., 37, L0885, doi: 10.1029/2010GL042761. [Link]

Knaff, J. A., J. P. Kossin, and M. DeMaria, 2003: Annular hurricanes. Weather Forecast., 18, 204-223, doi: 10.1175/1520-0434(2003)018<0204:AH>2.0.CO;2. [Link]

Knaff, J. A., C. R. Sampson, and M. DeMaria, 2005: An operational statistical typhoon intensity prediction scheme for the western North Pacific. Weather Forecast., 20, 688-699, doi: 10.1175/WAF863.1. [Link]

Kossin, J. P. and M. D. Eastin, 2001: Two distinct regimes in the kinematic and thermodynamic structure of the hurricane eye and eyewall. J. Atmos. Sci., 58, 10791090, doi: 10.1175/1520-0469(2001)058<1079:TDRI TK>2.0.CO;2. [Link]

Kossin, J. P. and W. H. Schubert, 2001: Mesovortices, polygonal flow patterns, and rapid pressure falls in hurricane-like vortices. J. Atmos. Sci., 58, 2196-2209, doi: 10.1175/1520-0469(2001)058<2196:MPFPAR > 2.0.C O;2. [Link]

Kossin, J. P. and M. Sitkowski, 2009: An objective model for identifying secondary eyewall formation in hurricanes. Mon. Weather Rev., 137, 876-892, doi: 10.1175/ 2008MWR2701.1. [Link]

Montgomery, M. T. and R. J. Kallenbach, 1997: A theory for vortex Rossby-waves and its application to spiral bands and intensity changes in hurricanes. Q.J.R. Meteorol.Soc., 123, 435-465, doi: 10.1002/qj.49712353810. [Link]

Schubert, W. H., M. T. Montgomery, R. K. Taft, T. A. Guinn, S. R. Fulton, J. P. Kossin, and J. P. Edwards, 1999: Polygonal eyewalls, asymmetric eye contraction, and potential vorticity mixing in hurricanes. J. Atmos. Sci., 56, 1197-1223, doi: 10.1175/1520-0469(1999)056 $<1197$ :PEAECA > 2.0.CO;2. [Link]

Shapiro, L. J. and H. E. Willoughby, 1982: The response of balanced hurricanes to local sources of heat and mo- 
mentum. J. Atmos. Sci., 39, 378-394, doi: 10.1175/152 0-0469(1982)039<0378:TROBHT>2.0.CO;2. [Link]

Thiébaux, J., E. Rogers, W. Wang, and B. Katz, 2003: A new high-resolution blended real-time global sea surface temperature analysis. Bull. Amer. Meteorol. Soc., 84, 645-656, doi: 10.1175/BAMS-84-5-645. [Link]

Velden, C., B. Harper, F. Wells, J. L. Beven II, R. Zehr, T. Olander, M. Mayfield, C. C. Guard, M. Lander, R. Edson, L. Avila, A. Burton, M. Turk, A. Kikuchi, A. Christian, P. Caroff, and P. McCrone, 2006: The Dvorak tropical cyclone intensity estimation technique: A satellite-based method that has endured for over 30 years. Bull. Amer. Meteorol. Soc., 87, 1195-1210, doi: 10.1175/BAMS-87-9-1195. [Link]

Wang, Y., 2002: Vortex Rossby waves in a numerically simulated tropical cyclone. Part I: Overall structure, potential vorticity, and kinetic energy budgets. J. Atmos. Sci., 59, 1213-1238, doi: 10.1175/1520-0469(2002) $059<1213$ :VRWIAN>2.0.CO;2. [Link]

Wang, Y. and C. C. Wu, 2004: Current understanding of tropical cyclone structure and intensity changes - A review. Meteorol. Atmos. Phys., 87, 257-278, doi: 10.10 07/s00703-003-0055-6. [Link]

Willoughby, H. E., 1998: Tropical cyclone eye thermody- namics. Mon. Weather Rev., 126, 3053-3067, doi: 10. 1175/1520-0493(1998)126<3053:TCET>2.0.CO;2. [Link]

Willoughby, H. E. and P. G. Black, 1996: Hurricane Andrew in Florida: Dynamics of a disaster. Bull. Amer. Meteorol. Soc., 77, 543-549, doi: 10.1175/1520-0477 (1996)077<0543:HAIFDO>2.0.CO;2. [Link]

Willoughby, H. E., J. A. Clos, and M. G. Shoreibah, 1982: Concentric eye walls, secondary wind maxima, and the evolution of the hurricane vortex. J. Atmos. Sci., 39, 395-411, doi: 10.1175/1520-0469(1982)039<0395:CE WSWM>2.0.CO;2. [Link]

Wu, C. C. and Y. H. Kuo, 1999: Typhoons affecting Taiwan: Current understanding and future challenges. Bull. Amer. Meteorol. Soc., 80, 67-80, doi: 10.1175/15 20-0477(1999)080<0067:TATCUA>2.0.CO;2. [Link]

Wu, C. C., K. H. Chou, H. J. Cheng, and Y. Wang, 2003: Eyewall contraction, breakdown and reformation in a landfalling typhoon. Geophys. Res. Lett., 30, 1887, doi: 10.1029/2003GL017653. [Link]

Wu, C. C., H. J. Cheng, Y. Wang, and K. H. Chou, 2009: A numerical investigation of the eyewall evolution in a landfalling typhoon. Mon. Weather Rev., 137, 21-40, doi: 10.1175/2008MWR2516.1. [Link] 